\title{
Oscillation Results for a Class of Nonlinear Fractional Order Difference Equations with Damping Term
}

\author{
A. George Maria Selvam $\mathbb{D}^{1},{ }^{1}$ Jehad Alzabut $\mathbb{D},{ }^{2}$ Mary Jacintha, ${ }^{1}$ and Abdullah Özbekler ${ }^{3}$ \\ ${ }^{1}$ Department of Mathematics, Sacred Heart College, Tirupattur, -635601 Tamil Nadu, India \\ ${ }^{2}$ Department of Mathematics and General Sciences, Prince Sultan University, Riyadh -11586, Saudi Arabia \\ ${ }^{3}$ Department of Mathematics, Atilim University, 06830, Incek, Ankara, Turkey
}

Correspondence should be addressed to Jehad Alzabut; jalzabut@psu.edu.sa

Received 17 February 2020; Accepted 4 May 2020; Published 1 June 2020

Guest Editor: Lishan Liu

Copyright $\odot 2020$ A. George Maria Selvam et al. This is an open access article distributed under the Creative Commons Attribution License, which permits unrestricted use, distribution, and reproduction in any medium, provided the original work is properly cited.

The paper studies the oscillation of a class of nonlinear fractional order difference equations with damping term of the form $\Delta\left[\psi(\lambda) z^{\eta}(\lambda)\right]+p(\lambda) z^{\eta}(\lambda)+q(\lambda) F\left(\sum_{s=\lambda}^{\lambda-1+\mu}(\lambda-s-1)^{(-\mu)} y(s)\right)=0$, where $z(\lambda)=a(\lambda)+b(\lambda) \Delta^{\mu} y(\lambda)$, $\Delta^{\mu}$ stands for the fractional difference operator in Riemann-Liouville settings and of order $\mu, 0<\mu \leq 1$, and $\eta \geq 1$ is a quotient of odd positive integers and $\lambda \in \mathbb{N}_{\lambda_{0}+1-\mu}$. New oscillation results are established by the help of certain inequalities, features of fractional operators, and the generalized Riccati technique. We verify the theoretical outcomes by presenting two numerical examples.

\section{Introduction and Background}

The objective of this paper is to provide oscillation theorems for the equation

$$
\begin{gathered}
\Delta\left[\psi(\lambda) z^{\eta}(\lambda)\right]+p(\lambda) z^{\eta}(\lambda)+q(\lambda) F \\
\cdot\left(\sum_{s=\lambda_{0}}^{\lambda-1+\mu}(\lambda-s-1)^{(-\mu)} y(s)\right)=0,
\end{gathered}
$$

where $\lambda \in \mathbb{N}_{\lambda_{0}+1-\mu}, \eta>0$ is a quotient of odd positive integers, $\Delta^{\mu}$ is the fractional difference operator in the sense of Riemann-Liouville (RL) and of order $\mu, 0<\mu \leq 1$, and

$$
z(\lambda)=a(\lambda)+b(\lambda) \Delta^{\mu} y(\lambda) .
$$

The $\mu^{\text {th }}$ fractional sum for $\mu>0$, (see [1]) is defined by

$$
\Delta_{a}^{-\mu} f(\lambda)=\frac{1}{\Gamma(\mu)} \sum_{s=a}^{\lambda-\mu}(\lambda-s-1)^{(\mu-1)} f(s), \quad \lambda \in \mathbb{N}_{a+\mu},
$$

where the fractional sum $\Delta^{-\mu}$ is defined from $\mathbb{N}_{a}$ to $\mathbb{N}_{a+\mu}$, $f(s)$ is defined for $s \equiv a \bmod (1)$ and $\Delta_{a}^{-\mu} f(\lambda)$ is defined for $\lambda \equiv(a+\mu) \bmod (1)$. The falling function is

$$
\lambda^{(\mu)}=\frac{\Gamma(\lambda+1)}{\Gamma(\lambda+1-\mu)} .
$$

where $\Gamma$ is the Gamma function, given by

$$
\Gamma(t):=\int_{0}^{\infty} s^{t-1} e^{-s} d s,
$$

for $t \in \mathbb{R}_{+}:=(0, \infty)$.

Let $\mu$ and $N$ be positive integers such that $N=\lceil\mu\rceil$, namely, $\mu \in(N-1, N)$. Set $v=N-\mu$. Then, $\mu^{\text {th }}$ fractional difference (see [2]) is defined as

$$
\Delta^{\mu} f(\lambda)=\Delta^{N-v} f(\lambda)=\Delta^{N} \Delta^{-v} f(\lambda) .
$$

The following conditions are assumed to hold throughout this work: 
(i) $\left(H_{1}\right) \psi(\lambda) \in C\left(\left[t_{0}, \infty\right)\right), p(\lambda) \in C\left(\left[t_{0}, \infty\right)\right)$, and $q(\lambda)$ $\in C\left(\left[t_{0}, \infty\right)\right)$ are positive sequences with $\psi(\lambda)>p(\lambda)$

(ii) $\left(H_{2}\right)$

$$
\Delta\left[\frac{a(\lambda)}{b(\lambda)}\right] \neq 0
$$

for $\lambda \in\left[\lambda_{0}, \infty\right)$ and

$$
\lim _{\lambda \rightarrow \infty} \sum_{k=\lambda_{0}}^{\lambda-1}\left[\frac{a(k)}{b(k)}\right]<\infty
$$

(iii) $\left(H_{3}\right) a(\lambda)$ is a nonnegative sequence on $\left[\lambda_{0}, \infty\right)$ for some $\lambda_{0}>0$. There exists a $n_{0}>0$ such that $b(\lambda) \geq$ $n_{0}$ for $\lambda \in\left[\lambda_{0}, \infty\right)$

(iv) $\left(\mathrm{H}_{4}\right) \mathrm{F}$ is a monotone decreasing function satisfying $\Psi F(\Psi)>0$ such that

$$
\frac{F(\Psi)}{\Psi^{\eta}} \geq L>0 \text { for } \Psi \neq 0
$$

A nontrivial solution of Eq. (1) is called oscillatory if it has arbitrarily large zeros. Otherwise, it is called nonoscillatory. Eq. (1) is called oscillatory if all its solutions are oscillatory.

In the literature, Wang et al. [3] extended some oscillation results from integer-order differential equation to the fractional-order differential equation

$$
\mathfrak{D}_{a}^{\alpha} x(t)+q(t) f(x(t))=0 ; \quad t \in[a,+\infty), a>0,
$$

where $\mathfrak{D}_{a}^{\alpha}$ denotes the standard RL differential operator of order $\alpha$ with $0<\alpha \leq 1, q(t)$ is a positive real-valued function, $f(\cdot):[0, \infty) \longrightarrow[0, \infty)$ is a continuous function satisfying

$$
\frac{f(u)}{u} I^{\alpha-2} \geq K>0
$$

and $I^{2-\alpha}$ denotes RL integral operator. Xiang et al. [4] investigated conditions for oscillation of fractional-order differential equation

$$
\left[a(t) w^{\gamma}(t)\right]-b(\lambda) f\left(\int_{t}^{\infty}(s-t)^{-\alpha} x(s) \mathrm{d} s\right)=0 ; \quad t \geq t_{0}>0
$$

where $w(t)=p(t)+q(t)\left(\mathfrak{D}_{-}^{\alpha} x\right)(t), \quad \alpha \in(0,1)$ is a fractional-order, $\gamma>0$ is a quotient of odd positive integers, and $\left(\mathfrak{D}_{-}^{\alpha} x\right)$ is the RL right-sided fractional derivative of order $\alpha$ of $x$ defined by

$$
\left(\mathfrak{D}_{-}^{\alpha} x\right)(t):=\frac{-1}{\Gamma(1-\alpha)} \frac{\mathrm{d}}{\mathrm{d} t} \int_{t}^{\infty}(s-t)^{-\alpha} x(s) \mathrm{d} s,
$$

for $t \in \mathbb{R}_{+}$.
In this paper and motivated by the above work, we intend to carry forward the oscillation results from fractional differential Eq. (12) to the fractional difference Eq. (1). Moreover, we consider Eq. (1) under a damping term.

Fractional difference calculus is evolving as a powerful tool for studying problems in many fields such as biology, mechanics, control systems, ecology, electrical networks, electrochemical of corrosion, chemical physics, optics, and signal processing, economics and so forth (see [5-12]) and the references therein. Basic definitions and properties of fractional difference calculus were presented by Goodrich and Peterson [1]. In addition, there are other research works dealing with fractional difference equations which have helped to build up some of the basic theory in this area (see [2, 13-15]).

Fractional difference equations are applied to model physical processes which vary with time and space and its nonlocal property enables to model systems with memory effect. The study of the qualitative analysis of these equations has gained momentum in recent years and thus numerous publications have been reviled [16-20].

Of late, the investigation of the oscillation of solutions for fractional order difference equations has accelerated with several articles (see [21-29]). In what follows, we state some lemmas and preliminaries that will contribute in proving our main results.

Definition 1 (see [2]). For any $\mu \geq 0$, the falling factorial is known as

$$
s^{(\mu)}=\frac{\Gamma(s+1)}{\Gamma(s+1-\mu)},
$$

where $\Gamma$ is as in (5).

Lemma 2 (see [23]). Let $y(\lambda)$ be a solution of (1) and

$$
\Psi(\lambda)=\sum_{s=\lambda_{0}}^{\lambda-1+\mu}(\lambda-s-1)^{(-\mu)} y(s)
$$

then

$$
\Delta \Psi(\lambda)=\Gamma(1-\mu) \Delta^{\mu} y(\lambda)
$$

Lemma 3 (see [30]). The product and quotient rules of the difference operator $\Delta$ are defined to be

$$
\Delta[x(\lambda) y(\lambda)]=\Delta x(\lambda) y(\lambda+1)+x(\lambda) \Delta y(\lambda)
$$

and

$$
\Delta\left[\frac{x(\lambda)}{y(\lambda)}\right]=\frac{\Delta x(\lambda) y(\lambda)-x(\lambda) \Delta y(\lambda)}{y(\lambda) y(\lambda+1)}
$$

where $\Delta x(\lambda)=x(\lambda+1)-x(\lambda)$. 
Lemma 4 (see [31]). Let $\eta \geq 1$ be a quotient of two positive odd integers. If

$$
\Psi(\lambda+1)>\Psi(\lambda)>0(\Psi(\lambda+1)<\Psi(\lambda)<0),
$$

then

$$
\Delta \Psi^{\eta}(\lambda) \geq(\Delta \Psi(\lambda))^{\eta}\left(\Delta \Psi^{\eta}(\lambda) \leq(\Delta \Psi(\lambda))^{\eta}\right) .
$$

Lemma 5 (see [32]). Let $\xi, \zeta \in \mathbb{R}$ with $\zeta>0$. Then, the inequality

$$
\xi X-\zeta X^{2} \leq \frac{\xi^{2}}{4 \zeta}
$$

holds for all $X \in \mathbb{R}$.

\section{Main Results}

Herein, new oscillation theorems for Eq. (1) are established by using mathematical inequalities, the properties of RL sum and difference operators, and the generalized Riccati technique.

Define the sequence

$$
u(\lambda)=\prod_{s=\lambda_{0}}^{\lambda-1} \frac{\psi(s)}{\psi(s)-p(s)} .
$$

Then $u(\lambda)>0$,

$$
u(\lambda+1)=\frac{\psi(\lambda)}{\psi(\lambda)-p(\lambda)} u(\lambda)
$$

and hence

$$
\Delta u(\lambda)=u(\lambda+1)-u(\lambda)=\frac{p(\lambda)}{\psi(\lambda)-p(\lambda)} u(\lambda) .
$$

Theorem 6. Assume that $\left(H_{1}\right)-\left(H_{4}\right)$ hold. If

$$
\lim _{\lambda \rightarrow \infty} \sum_{v=\lambda_{0}}^{\lambda-1}\left[\frac{1}{u(v) \psi(v)}\right]^{1 / \eta}=\infty
$$

and

$$
\lim _{\lambda \rightarrow \infty} \sum_{v=\lambda_{2}}^{\lambda-1}\left[L u(v) q(v)-\frac{(\Delta u(v))^{2} \psi(v+1) N^{\eta}}{4 u(v+1)}\right]=\infty,
$$

then Eq. (1) is oscillatory.

Proof. Suppose that $y(\lambda)$ is a nonoscillatory solution of Eq. (1). Without loss of generality, we may assume that $y(\lambda)$ is an eventually positive solution of Eq. (1). Then, there exits $\lambda_{1} \in\left[\lambda_{0}, \infty\right)$ such that $y(\lambda)>0, \Psi(\lambda)>0$ for $\lambda \in\left[\lambda_{1}, \infty\right)$, where $\Psi(\lambda)$ is defined in Lemma 2. Considering the assumption $\left(H_{4}\right)$ and using Eq. (1), we get

$$
\Delta\left[\psi(\lambda) z^{\eta}(\lambda)\right]+p(\lambda) z^{\eta}(\lambda)=-q(\lambda) F(\Psi(\lambda)),
$$

or

$$
\Delta\left[\psi(\lambda) z^{\eta}(\lambda)\right]+p(\lambda) z^{\eta}(\lambda) \leq-L q(\lambda) \Psi^{\eta}(\lambda),
$$

where $z(\lambda)$ is defined in (2). Hence, we proceed from (22) and (17) to

$$
\begin{aligned}
\Delta\left[u(\lambda) \psi(\lambda) z^{\eta}(\lambda)\right]= & u(\lambda+1) \Delta\left[\psi(\lambda) z^{\eta}(\lambda)\right] \\
& +\Delta u(\lambda) \psi(\lambda) z^{\eta}(\lambda) \\
= & u(\lambda+1) \Delta\left[\psi(\lambda) z^{\eta}(\lambda)\right] \\
& +u(\lambda) \frac{p(\lambda)}{\psi(\lambda)-p(\lambda)} \psi(\lambda) z^{\eta}(\lambda) \\
= & u(\lambda+1) \Delta\left[\psi(\lambda) z^{\eta}(\lambda)\right] \\
& +u(\lambda+1) \frac{\psi(\lambda)-p(\lambda)}{\psi(\lambda)} \\
& \cdot \frac{p(\lambda)}{\psi(\lambda)-p(\lambda)} \psi(\lambda) z^{\eta}(\lambda) \\
= & u(\lambda+1)\left[\Delta\left[\psi(\lambda) z^{\eta}(\lambda)\right]+p(\lambda) z^{\eta}(\lambda)\right] \\
\leq & -L u(\lambda+1) q(\lambda) \Psi^{\eta}(\lambda)<0 .
\end{aligned}
$$

Then, $u(\lambda) \psi(\lambda) z^{\eta}(\lambda)$ is strictly decreasing on $\left[\lambda_{1}, \infty\right)$ and is eventually of constant sign. Since $u(\lambda)>0, \psi(\lambda)>0$, and $\eta>0$ is a quotient of odd positive integers, we observe that $z(\lambda)$ is eventually of constant sign.

First, we show that

$$
z(\lambda)>0 \text { for } \lambda \in\left[\lambda_{1}, \infty\right) .
$$

If not, then there exits $\lambda_{2} \geq \lambda_{1}$ such that $z\left(\lambda_{2}\right)<0$, and we obtain

$$
u(\lambda) \psi(\lambda) z^{\eta}(\lambda)<u\left(\lambda_{2}\right) \psi\left(\lambda_{2}\right) z^{\eta}\left(\lambda_{2}\right)=d<0,
$$

which implies

$$
z^{\eta}(\lambda)<\frac{d}{u(\lambda) \psi(\lambda)}<0
$$

for $\lambda \in\left[\lambda_{2}, \infty\right)$ that is

$$
z(\lambda)<\left[\frac{d}{u(\lambda) \psi(\lambda)}\right]^{1 / \eta}<0
$$

So we arrive at that $z(\lambda)<0$ on $\left[\lambda_{2}, \infty\right)$. Hence for $\lambda$ $\epsilon\left[\lambda_{2}, \infty\right)$, we get 


$$
\begin{aligned}
\frac{a(\lambda)}{b(\lambda)}+\Delta^{\mu} y(\lambda) & <\frac{z(\lambda)}{b(\lambda)}<\frac{1}{b(\lambda)}\left[\frac{d}{u(\lambda) \psi(\lambda)}\right]^{1 / \eta} \\
& <\frac{1}{n_{0}}\left[\frac{d}{u(\lambda) \psi(\lambda)}\right]^{1 / \eta} .
\end{aligned}
$$

From (16), we have

$$
\frac{a(\lambda)}{b(\lambda)}+\frac{\Delta \Psi(\lambda)}{\Gamma(1-\mu)}=\frac{a(\lambda)}{b(\lambda)}+\Delta^{\alpha} y(\lambda)<\frac{1}{n_{0}}\left[\frac{d}{u(\lambda) \psi(\lambda)}\right]^{1 / \eta},
$$

and summing from $\lambda_{2}$ to $\lambda-1$, we obtain

$$
\sum_{v=\lambda_{2}}^{\lambda-1} \Delta \Psi(v)<\Gamma(1-\mu) \sum_{v=\lambda_{2}}^{\lambda-1}\left[\frac{1}{n_{0}}\left[\frac{d}{u(v) \psi(v)}\right]^{\frac{1}{\eta}}-\frac{a(v)}{b(v)}\right],
$$

and hence we have

$$
\Psi(\lambda)<\Psi\left(\lambda_{2}\right)+\Gamma(1-\mu) \sum_{v=\lambda_{2}}^{\lambda-1}\left[\frac{1}{n_{0}}\left[\frac{d}{u(v) \psi(v)}\right]^{1 / \eta}-\frac{a(v)}{b(v)}\right] .
$$

Now, by letting $\lambda \longrightarrow \infty$, we get

$$
\begin{aligned}
\lim _{\lambda \rightarrow \infty} \Psi(\lambda) & <\lim _{\lambda \rightarrow \infty}\left\{\Psi\left(\lambda_{2}\right)+\Gamma(1-\mu) \sum_{v=\lambda_{2}}^{\lambda-1}\left[\frac{1}{n_{0}}\left[\frac{d}{u(v) \psi(v)}\right]^{1 / \eta}-\frac{a(v)}{b(v)}\right]\right\} \\
& <\Psi\left(\lambda_{2}\right)+\Gamma(1-\mu) \sum_{v=\lambda_{2}}^{\infty}\left[\frac{1}{n_{0}}\left[\frac{d}{u(v) \psi(v)}\right]^{1 / \eta}-\frac{a(v)}{b(v)}\right]=-\infty
\end{aligned}
$$

which contradicts that $\Psi(\lambda)>0, \lambda \in\left[\lambda_{1}, \infty\right)$. Therefore $z$ $(\lambda)>0$ for $\lambda \in\left[\lambda_{2}, \infty\right)$.

Now, since

$$
\frac{z(\lambda)}{b(\lambda)}=\frac{a(\lambda)}{b(\lambda)}+\Delta^{\mu} y(\lambda)=\frac{a(\lambda)}{b(\lambda)}+\frac{\Delta \Psi(\lambda)}{\Gamma(1-\mu)},
$$

and we have

$$
\frac{\Delta \Psi(\lambda)}{\Gamma(1-\mu)}=\frac{z(\lambda)-a(\lambda)}{b(\lambda)}<\frac{z(\lambda)-a(\lambda)}{n_{0}}<\frac{z(\lambda)}{n_{0}},
$$

from (16), we obtain

$$
\Delta \Psi(\lambda)<\frac{z(\lambda)}{n_{0}}
$$

Define the generalized Riccati function

$$
w(\lambda)=u(\lambda) \frac{\psi(\lambda) z^{\eta}(\lambda)}{\Psi^{\eta}(\lambda)} ; \quad \lambda \in\left[\lambda_{1}, \infty\right) .
$$

It is clear that $w(\lambda)>0$. Using (17) and (18) for $\lambda$ $\epsilon\left[\lambda_{0}, \infty\right)$, we have

$$
\begin{aligned}
\Delta w(\lambda)= & \Delta u(\lambda) \frac{\psi(\lambda+1) z^{\eta}(\lambda+1)}{\Psi^{\eta}(\lambda+1)} \\
& +u(\lambda) \Delta\left[\frac{\psi(\lambda) z^{\eta}(\lambda)}{\Psi^{\eta}(\lambda)}\right]=\Delta u(\lambda) \frac{w(\lambda+1)}{u(\lambda+1)} \\
& +u(\lambda)\left[\frac{\Delta\left\{\psi(\lambda) z^{\eta}(\lambda)\right\} \Psi^{\eta}(\lambda)-\psi(\lambda) z^{\eta}(\lambda) \Delta \Psi^{\eta}(\lambda)}{\Psi^{\eta}(\lambda) \Psi^{\eta}(\lambda+1)}\right] \\
= & \Delta u(\lambda) \frac{w(\lambda+1)}{u(\lambda+1)}+u(\lambda) \frac{\Delta\left\{\psi(\lambda) z^{\eta}(\lambda)\right\}}{\Psi^{\eta}(\lambda+1)} \\
& -u(\lambda) \frac{\psi(\lambda) z^{\eta}(\lambda)}{\Psi^{\eta}(\lambda) \Psi^{\eta}(\lambda+1)} \Delta \Psi^{\eta}(\lambda)=\Delta u(\lambda) \frac{w(\lambda+1)}{u(\lambda+1)} \\
& -u(\lambda)\left[\frac{p(\lambda) z^{\eta}(\lambda)+q(\lambda) f(\Psi(\lambda))}{\Psi^{\eta}(\lambda+1)}\right] \\
& -\frac{u(\lambda) \psi(\lambda) z^{\eta}(\lambda)}{\Psi^{\eta}(\lambda) \Psi^{\eta}(\lambda+1)} \Delta \Psi^{\eta}(\lambda)=\Delta u(\lambda) \frac{w(\lambda+1)}{u(\lambda+1)} \\
& -\frac{u(\lambda) p(\lambda) z^{\eta}(\lambda)}{\Psi^{\eta}(\lambda+1)}-\frac{u(\lambda) q(\lambda) f(\Psi(\lambda))}{\Psi^{\eta}(\lambda+1)} \\
& -u(\lambda) \frac{\psi(\lambda) z^{\eta}(\lambda)}{\Psi^{\eta}(\lambda) \Psi^{\eta}(\lambda+1)} \Delta \Psi^{\eta}(\lambda) .
\end{aligned}
$$

Since

$$
\frac{u(\lambda) \psi(\lambda) z^{\eta}(\lambda)}{\Psi^{\eta}(\lambda) \Psi^{\eta}(\lambda+1)}>0
$$

and $\Delta \Psi(\lambda)>0$, then $\Psi(\lambda+1)-\Psi(\lambda)>0$; it follows from $\left(H_{4}\right)$ that

$$
\frac{f(\Psi(\lambda))}{\Psi^{\eta}(\lambda+1)}>\frac{f(\Psi(\lambda+1))}{\Psi^{\eta}(\lambda+1)} \geq L,
$$

and $\Delta \Psi^{\eta}(\lambda)>(\Delta \Psi(\lambda))^{\eta}$ by Lemma 4 .

Using the fact that $u(\lambda) \psi(\lambda) z^{\eta}(\lambda)$ is strictly decreasing, we arrive at

$$
\frac{u(\lambda) \psi(\lambda) z^{\eta}(\lambda)}{\Psi^{\eta}(\lambda) \Psi^{\eta}(\lambda+1)} \Delta \Psi^{\eta}(\lambda) \geq \frac{u(\lambda) \psi(\lambda) z^{\eta}(\lambda)}{\Psi^{\eta}(\lambda) \Psi^{\eta}(\lambda+1)}(\Delta \Psi(\lambda))^{\eta}
$$

Also we have

$$
(\Delta \Psi(\lambda))^{\eta}<\left[\frac{z(\lambda)}{n_{0}}\right]^{\eta}
$$

from (41). 
Now substituting (45), (46), and (47) in (43), we get

$$
\begin{aligned}
\Delta w(\lambda)< & \Delta u(\lambda) \frac{w(\lambda+1)}{u(\lambda+1)}-L u(\lambda) q(\lambda) \\
& -\frac{u(\lambda) \psi(\lambda) z^{\eta}(\lambda) \frac{z^{\eta}(\lambda)}{\Psi^{\eta}(\lambda) \Psi^{\eta}(\lambda+1)}}{n_{0}^{\eta}} \\
< & \Delta u(\lambda) \frac{w(\lambda+1)}{u(\lambda+1)}-L u(\lambda) q(\lambda) \\
& -\frac{u(\lambda+1) \psi(\lambda+1) z^{\eta}(\lambda+1)}{\Psi^{\eta}(\lambda+1) \Psi^{\eta}(\lambda+1)} \frac{z^{\eta}(\lambda+1)}{n_{0}^{\eta}} \\
< & \Delta u(\lambda) \frac{w(\lambda+1)}{u(\lambda+1)}-L u(\lambda) q(\lambda) \\
& -\frac{\left[u(\lambda+1) \psi(\lambda+1) z^{\eta}(\lambda+1)\right]^{2}}{u(\lambda+1) \psi(\lambda+1)\left[\Psi^{\eta}(\lambda+1)\right]^{2} n_{0}^{\eta}} \\
< & \Delta u(\lambda) \frac{w(\lambda+1)}{u(\lambda+1)}-L u(\lambda) q(\lambda) \\
& -\left[\frac{u(\lambda+1) \psi(\lambda+1) z^{\eta}(\lambda+1)}{\Psi^{\eta}(\lambda+1)}\right]^{2} \\
& \times \frac{1}{u(\lambda+1) \psi(\lambda+1) n_{0}^{\eta}<\Delta u(\lambda) \frac{w(\lambda+1)}{u(\lambda+1)}} \\
& -L u(\lambda) q(\lambda)-\frac{1}{u(\lambda+1) \psi(\lambda+1) n_{0}^{\eta}} w^{2}(\lambda+1) .
\end{aligned}
$$

Using (48) and (21) with $X=w(\lambda+1), \quad a=\Delta u(\lambda) / u$ $(\lambda+1)$, and

$$
b=\frac{1}{u(\lambda+1) \psi(\lambda+1) n_{0}^{\eta}}
$$

we obtain

$$
\begin{aligned}
\Delta w(\lambda) & <-L u(\lambda) q(\lambda)+\frac{(\Delta u(\lambda))^{2} \psi(\lambda+1) u(\lambda+1) n_{0}^{\eta}}{4(u(\lambda+1))^{2}} \\
& =-L u(\lambda) q(\lambda)+\frac{(\Delta u(\lambda))^{2} \psi(\lambda+1) n_{0}^{\eta}}{4 u(\lambda+1)}
\end{aligned}
$$

and hence

$$
\left[\operatorname{Lu}(\lambda) q(\lambda)-\frac{(\Delta u(\lambda))^{2} \psi(\lambda+1) n_{0}^{\eta}}{4 u(\lambda+1)}\right]<-\Delta w(\lambda) .
$$

Summing up (51) from $\lambda_{2}$ to $\lambda-1$, we have

$$
\begin{gathered}
\sum_{v=\lambda_{2}}^{\lambda-1}\left[L u(v) q(v)-\frac{(\Delta u(v))^{2} \psi(v+1) n_{0}^{\eta}}{4 u(v+1)}\right]<-\sum_{v=\lambda_{2}}^{\lambda-1} \Delta w(v) \\
=-\left[w(\lambda)-w\left(\lambda_{2}\right)\right]=w\left(\lambda_{2}\right)-w(\lambda),
\end{gathered}
$$

and hence

$$
\sum_{v=\lambda_{2}}^{\lambda-1}\left[L u(v) q(v)-\frac{(\Delta u(v))^{2} \psi(v+1) n_{0}^{\eta}}{4 u(v+1)}\right]<w\left(\lambda_{2}\right)
$$

Letting $\lambda \longrightarrow \infty$,

$$
\lim _{\lambda \rightarrow \infty} \sum_{v=\lambda_{2}}^{\lambda-1}\left[L u(v) q(v)-\frac{(\Delta u(v))^{2} \psi(v+1) n_{0}^{\eta}}{4 u(v+1)}\right]<w\left(\lambda_{2}\right)<\infty
$$

which contradicts with (26).

Theorem 7. Assume that (25)and (26) hold and there exists a positive sequence $H(t, s)$ such that

(1) $H(\lambda, \lambda)=0$ for $\lambda \geq \lambda_{0} ; H(\lambda, v)>0$ for $\lambda>v \geq \lambda_{0}$

(2) $\Delta_{2} H(\lambda, v)=H(\lambda, v+1)-H(\lambda, v)<0$ for $\lambda>v \geq \lambda_{0}$

If

$$
\begin{aligned}
\lim _{\lambda \rightarrow \infty} \frac{1}{H\left(\lambda, \lambda_{0}\right)} \sum_{v=\lambda_{0}}^{\lambda-1}[L u(v) q(v) H(\lambda, v) \\
\left.-\frac{h_{+}^{2}(\lambda, v) u(v+1) \psi(v+1) n_{0}^{\eta}}{4 H(\lambda, v)}\right]=\infty,
\end{aligned}
$$

then Eq. (1) is oscillatory, where $u(\lambda)$ is defined in Theorem 6 and

$$
h_{+}(\lambda, v)=\Delta_{2} H(\lambda, v)+\frac{\Delta u(v) H(\lambda, v)}{u(v+1)}
$$

Proof. Suppose that $y(\lambda)$ is a nonoscillatory solution of (1). Without loss of generality, we may assume that $y(\lambda)$ is an eventually positive solution of (1). Then, there exists $\lambda_{1}$ $\in\left[\lambda_{0}, \infty\right)$ such that $y(\lambda)>0, \Psi(\lambda)>0$ for $\lambda \in\left[\lambda_{1}, \infty\right)$. Proceeding as in proof of Theorem 6 we arrive at (48). Multiplying (48) by $H(\lambda, v)$ and summing up from $\lambda_{2}$ to $\lambda-1$, we obtain

$$
\begin{aligned}
& \sum_{v=\lambda_{2}}^{\lambda-1} \Delta w(v) H(\lambda, v)<\sum_{v=\lambda_{2}}^{\lambda-1} \frac{\Delta u(v) w(v+1) H(\lambda, v)}{u(v+1)} \\
& -\sum_{v=\lambda_{2}}^{\lambda-1} L u(v) q(v) H(\lambda, v) \\
& -\sum_{v=\lambda_{2}}^{\lambda-1} \frac{H(\lambda, v) w^{2}(v+1)}{u(v+1) \psi(v+1) n_{0}^{\eta}}
\end{aligned}
$$


Using summation by parts formula, we get

$$
\begin{aligned}
-\sum_{v=\lambda_{2}}^{\lambda-1} \Delta w(v) H(\lambda, v) & =-\left.H(\lambda, v) w(\lambda)\right|_{v=\lambda_{2}} ^{v=\lambda-1}+\sum_{v=\lambda_{2}}^{\lambda-1} w(\lambda+1) \Delta_{2} H(\lambda, v) \\
& =H\left(\lambda, \lambda_{2}\right) w\left(\lambda_{2}\right)+\sum_{v=\lambda_{2}}^{\lambda-1} w(v+1) \Delta_{2} H(\lambda, v) .
\end{aligned}
$$

Hence, from (57)

$$
\begin{array}{rl}
\sum_{v=\lambda_{2}}^{\lambda-1} L & u(v) q(v) H(\lambda, v)<H\left(\lambda, \lambda_{2}\right) w\left(\lambda_{2}\right) \\
& +\sum_{v=\lambda_{2}}^{\lambda-1} w(v+1) \Delta_{2} H(\lambda, v) \\
& +\sum_{v=\lambda_{2}}^{\lambda-1} \frac{\Delta u(v) w(v+1) H(\lambda, v)}{u(v+1)} \\
& -\sum_{v=\lambda_{2}}^{\lambda-1} \frac{H(\lambda, v) w^{2}(v+1)}{u(v+1) \psi(v+1) n_{0}^{\eta}} \\
< & \sum_{v=\lambda_{2}}^{\lambda-1}\left[\Delta_{2} H(\lambda, v)+\frac{\Delta u(v) H(\lambda, v)}{u(v+1)}\right] w(v+1) \\
& -\sum_{v=\lambda_{2}}^{\lambda-1} \frac{H(\lambda, v) w^{2}(v+1)}{u(v+1) \psi(v+1) n_{0}^{\eta}}+H\left(\lambda, \lambda_{2}\right) w\left(\lambda_{2}\right) \\
< & H\left(\lambda, \lambda_{2}\right) w\left(\lambda_{2}\right)+\sum_{v=\lambda_{2}}^{\lambda-1}\left[h_{+}(\lambda, v) w(v+1)\right. \\
& -\frac{H(\lambda, v) w^{2}(v+1)}{\left.u(v+1) \psi(v+1) n_{0}^{\eta}\right] .}
\end{array}
$$

Now by using (21) with $X=w(v+1), \xi=h_{+}(\lambda, v)$ and

$$
\zeta=\frac{H(\lambda, v)}{u(v+1) \psi(v+1) n_{0}^{\eta}}
$$

we obtain

$$
\begin{aligned}
& \sum_{v=\lambda_{2}}^{\lambda-1}\left[L u(v) q(v) H(\lambda, v)-\frac{h_{+}^{2}(\lambda, v) u(v+1) \psi(v+1) n_{0}^{\eta}}{4 H(\lambda, v)}\right] \\
& <H\left(\lambda, \lambda_{2}\right) w\left(\lambda_{2}\right)<H\left(\lambda, \lambda_{0}\right) w\left(\lambda_{2}\right),
\end{aligned}
$$

for $\lambda>\lambda_{2}>\lambda_{1}>\lambda_{0}$. Then

$$
\begin{aligned}
& \sum_{v=\lambda_{0}}^{\lambda-1} {\left[L u(v) q(v) H(\lambda, v)-\frac{\left.h_{+}^{2}(\lambda, v) u(v+1) \psi(v+1) n_{0}^{\eta}\right]}{4 H(\lambda, v)}\right] } \\
&= \sum_{v=\lambda_{0}}^{\lambda_{2}-1}\left[L u(v) q(v) H(\lambda, v)-\frac{\left.h_{+}^{2}(\lambda, v) u(v+1) \psi(v+1) n_{0}^{\eta}\right]}{4 H(\lambda, v)}\right] \\
&+\sum_{v=\lambda_{2}}^{\lambda-1}\left[L u(v) q(v) H(\lambda, v)-\frac{h_{+}^{2}(\lambda, v) u(v+1) \psi(v+1) n_{0}^{\eta}}{4 H(\lambda, v)}\right] \\
&< \sum_{v=\lambda_{0}}^{\lambda_{2}-1}\left[L u(v) q(v) H(\lambda, v)-\frac{h_{+}^{2}(\lambda, v) u(v+1) \psi(v+1) n_{0}^{\eta}}{4 H(\lambda, v)}\right] \\
&+H\left(\lambda, \lambda_{0}\right) w\left(\lambda_{2}\right)<H\left(\lambda, \lambda_{0}\right) \sum_{v=\lambda_{0}}^{\lambda_{2}-1} L u(v) q(v) \\
&+H\left(\lambda, \lambda_{0}\right) w\left(\lambda_{2}\right), \\
& \text { which } \operatorname{yields}<\sum_{v=\lambda_{0}}^{\lambda} \operatorname{Lu}(v) q(v)+w\left(\lambda_{2}\right) . \\
& \frac{1}{H\left(\lambda, \lambda_{0}\right)} \sum_{v=\lambda_{0}}^{\lambda-1}\left[L u(v) q(v) H(\lambda, v)-\frac{h_{+}^{2}(\lambda, v) u(v+1) \psi(v+1) n_{0}^{\eta}}{4 H(\lambda, v)}\right. \\
& \quad \lambda_{2}-1
\end{aligned}
$$

Taking limit as $\lambda \longrightarrow \infty$, we get

$$
\begin{aligned}
\lim _{\lambda \rightarrow \infty} & \frac{1}{H\left(\lambda, \lambda_{0}\right)} \sum_{v=\lambda_{0}}^{\lambda-1}\left[L u(v) q(v) H(\lambda, v)-\frac{h_{+}^{2}(\lambda, v) u(v+1) \psi(v+1) n_{0}^{\eta}}{4 H(\lambda, v)}\right] \\
& <\sum_{v=\lambda_{0}}^{\lambda_{2}-1} \operatorname{Lu}(v) q(v)+w\left(\lambda_{2}\right)<\infty
\end{aligned}
$$

which contradicts with (55).

Next, we consider the condition

$$
\lim _{\lambda \rightarrow \infty} \sum_{v=\lambda_{0}}^{\lambda-1}\left[\frac{1}{u(v) \psi(v)}\right]^{1 / \eta}<\infty
$$

which implies that (25) does not hold. Under this condition, we have the following result.

Theorem 8. Assume that $\left(H_{1}\right)-\left(H_{4}\right),(26)$, and (65) hold. If

$$
\lim _{\lambda \rightarrow \infty} \sum_{s=\lambda_{2}}^{\lambda-1}\left[\left[\frac{1}{u(s) \psi(s)} \sum_{v=s}^{\infty} u(v+1) q(v)\right]^{1 / \eta}-\frac{a(s)}{b(s)}\right]=\infty,
$$

then every solution $y(\lambda)$ is oscillatory or satisfies $\lim _{\lambda \rightarrow \infty} \Psi$ $(\lambda)=0$, where $\Psi(\lambda)$ is defined in Lemma 2 . 
Proof. Assume that $y(\lambda)$ is a nonoscillatory solution of (1). Without loss of generality, assume that $y(\lambda)$ is eventually a positive solution of (1). Proceeding like in the proof of Theorem 6 , we get that (28) holds. Then, there are two signs of $z$ $(\lambda)$. When $z(\lambda)>0$ is eventually positive, we conclude from the proof of Theorem 6 that equation (1) is oscillatory.

Next, assume that $z(\lambda)$ is eventually negative, then there exits $\lambda_{2}>\lambda_{0}$ such that $z(\lambda)<0$ for $\lambda \geq \lambda_{2}$. Since $z$ $(\lambda)=a(\lambda)+b(\lambda) \Delta^{\mu} y(\lambda)$, we have

$$
a(\lambda)+b(\lambda) \frac{\Delta \Psi(\lambda)}{\Gamma(1-\mu)}=z(\lambda)<0
$$

and hence

$$
\Delta \Psi(\lambda)<-\Gamma(1-\mu) \frac{a(\lambda)}{b(\lambda)}
$$

On the other hand, Since $\left(H_{2}\right)$ holds, we get

$$
\lim _{\lambda \rightarrow \infty} \frac{a(\lambda)}{b(\lambda)}=0
$$

Now, taking the limit of the both sides of (68) as $\lambda$ tends to $\infty$, we get

$$
\lim _{\lambda \rightarrow \infty} \Delta \Psi(\lambda) \leq 0
$$

Since $\Psi(\lambda)>0$ for $\lambda \in\left[\lambda_{1}, \infty\right)$, we have

$$
\lim _{\lambda \rightarrow \infty} \Psi(\lambda)=\beta \geq 0
$$

Claim that $\beta=0$. If not, then $\Psi(\lambda) \geq \beta$ for $\lambda \in\left[\lambda_{2}, \infty\right)$. Now we have

$$
\begin{aligned}
\Delta\left[u(\lambda) \psi(\lambda) z^{\eta}(\lambda)\right] & \leq-L u(\lambda+1) q(\lambda) \Psi^{\eta}(\lambda) \\
& \leq-L u(\lambda+1) q(\lambda) \beta^{\eta}
\end{aligned}
$$

by (29). Summing up from $\lambda$ to $\infty$, we have

$$
\begin{aligned}
\sum_{v=\lambda}^{\infty} \Delta\left[u(v) \psi(v) z^{\eta}(v)\right] & =\lim _{v \rightarrow \infty} u(v) \psi(v) z^{\eta}(v)-u(\lambda) \psi(\lambda) z^{\eta}(\lambda) \\
& \leq-\beta^{\eta} L \sum_{v=\lambda}^{\infty} u(v+1) q(v),
\end{aligned}
$$

which yields

$$
\begin{aligned}
u(\lambda) \psi(\lambda) z^{\eta}(\lambda) & \geq \lim _{v \rightarrow \infty} u(v) \psi(v) z^{\eta}(v)+\beta^{\eta} L \sum_{v=\lambda}^{\infty} u(v+1) q(v) \\
& \geq \beta^{\eta} L \sum_{v=\lambda}^{\infty} u(v+1) q(v),
\end{aligned}
$$

and hence

$\frac{a(\lambda)}{b(\lambda)}+\frac{\Delta \Psi(\lambda)}{\Gamma(1-\mu)}=z(\lambda)>L^{1 / \eta} \beta\left[\frac{1}{u(\lambda) \psi(\lambda)} \sum_{v=\lambda}^{\infty} u(v+1) q(v)\right]^{1 / \eta}$

The last inequality above implies that

$\Delta \Psi(\lambda)>\Gamma(1-\mu)\left[L^{1 / \eta} \beta\left[\frac{1}{u(\lambda) \psi(\lambda)} \sum_{v=\lambda}^{\infty} u(v+1) q(v)\right]^{1 / \eta}-\frac{a(\lambda)}{b(\lambda)}\right]$

Summing up from $\lambda_{2}$ to $\lambda-1$, we get

$$
\begin{aligned}
& \Psi(\lambda)>\Psi\left(\lambda_{2}\right)+\Gamma(1-\mu) \\
& \cdot\left[L^{1 / \eta} \beta \sum_{s=\lambda_{2}}^{\lambda-1}\left[\left[\frac{1}{u(s) \psi(s)} \sum_{v=s}^{\infty} u(v+1) q(v)\right]^{1 / \eta}-\frac{a(s)}{b(s)}\right]\right] .
\end{aligned}
$$

Taking the limit of the both sides of the above inequality as $\lambda$ tends to $\infty$, we end up with

$$
\lim _{\lambda \rightarrow \infty} \Psi(\lambda)=\infty
$$

which contradicts to $\Psi(\lambda)>0$ for $\lambda \in\left[\lambda_{1}, \infty\right)$. Therefore, we obtain $\beta=0$, that is

$$
\lim _{\lambda \rightarrow \infty} \Psi(\lambda)=0
$$

\section{Applications}

Example 9. Consider the equation

$$
\Delta\left[\lambda^{2} x^{\eta}(\lambda)\right]+l x^{\eta}(\lambda)+\frac{1}{\lambda^{2}}\left(\sum_{s=\lambda_{0}}^{\lambda-1+\mu}(\lambda-s-1)^{-\mu} y(s)\right)^{-3}=0,
$$

where $\mu \in(0,1)$,

$$
x(\lambda)=e^{-\lambda}+\frac{1}{\lambda} \Delta^{\mu} y(\lambda), \quad \lambda \in \mathbb{N}_{2}=\{2,3,4, \cdots\},
$$

and that $\eta>0$ is a quotient of odd positive integers in $\mathbb{N}_{2}$. 
Comparing with Eq. (1), we get $\psi(\lambda)=\lambda^{2}, a(\lambda)=e^{-\lambda}, b$ $(\lambda)=1 / \lambda, p(\lambda)=\lambda, F(y)=y^{-3}, q(\lambda)=1 / \lambda^{2}, \eta=3, \lambda_{0}=2, n_{0}$ $=4, L=1 / 3$, and that

$$
\frac{f(y)}{y^{\eta}}=\frac{1}{y^{6}}>\varepsilon=L>0 \text { for } y \neq 0,
$$

where $\varepsilon$ is a certain positive number. It is clear that assumptions $\left(H_{1}\right)-\left(H_{4}\right)$ hold.

Further from (22), we have

$$
\begin{gathered}
u(\lambda)=\prod_{v=\lambda_{0}}^{\lambda-1} \frac{\psi(v)}{\psi(v)-p(v)}=\prod_{v=2}^{\lambda-1} \frac{v^{2}}{v^{2}-v}=\lambda-1 ; \\
\Delta u(\lambda)=u(\lambda+1)-u(\lambda)=1 ; \\
\Delta\left[\frac{a(\lambda)}{b(\lambda)}\right]=\Delta\left[\lambda e^{-\lambda}\right]=(\lambda+1) e^{-(\lambda+1)}(1-e)+e^{-\lambda} \neq 0 ; \\
\lim _{\lambda \rightarrow \infty} \sum_{v=\lambda_{0}}^{\lambda-1} \frac{a(v)}{b(v)}=\lim _{\lambda \rightarrow \infty} \sum_{v=1}^{\lambda-1} v e^{-v}<\infty ; \\
\lim _{\lambda \rightarrow \infty} \sum_{v=\lambda_{0}}^{\lambda-1}\left[\frac{1}{u(v) \psi(v)}\right]^{1 / \eta}=\lim _{\lambda \rightarrow \infty} \sum_{v=2}^{\lambda-1}\left[\frac{1}{v^{2}(v-1)}\right]^{1 / 3} \\
>\sum_{v=2}^{\infty} \frac{1}{v}=\infty ; \\
\lim _{\lambda \rightarrow \infty} \sum_{v=\lambda_{2}}^{\lambda-1}\left[L u(v) q(v)-\frac{(\Delta u(v))^{2} \psi(v+1) n_{0}^{\eta}}{4 u(v+1)}\right] \\
=\lim _{\lambda \rightarrow \infty} \sum_{v=2}^{\lambda-1}\left[\frac{(v-1)}{3 v^{2}}-\frac{16(v+1)^{2}}{v}\right]=\infty .
\end{gathered}
$$

Thus, conditions (25) and (26) are satisfied. Therefore, all solutions of (80) are oscillatory by Theorem 6 .

Example 10. Consider the equation

$\Delta\left[\frac{1}{\lambda} z^{\eta}(\lambda)\right]+\frac{1}{\lambda^{2}} z^{\eta}(\lambda)+\frac{1}{\lambda-1}\left(\sum_{s=\lambda_{0}}^{\lambda-1+\mu}(\lambda-s-1)^{-\mu} y(s)\right)^{-1}=0$,

where $\mu \in(0,1), z(\lambda)=\lambda^{2}+\lambda \Delta^{\mu} y(\lambda), \lambda \in \mathbb{N}_{2}$, and that $\eta>0$ is a quotient of odd positive integers in $\mathbb{N}_{2}$. Comparing with (1), we have $\psi(\lambda)=1 / \lambda, a(\lambda)=\lambda^{2}, b(\lambda)=\lambda, p(\lambda)=1$ $/ \lambda^{2}, F(y)=y^{-1}, q(\lambda)=1 /(\lambda-1), \eta=1, \lambda_{0}=2, n_{0}=2, L=1$ 12 , and that

$$
\frac{f(y)}{y^{\eta}}=\frac{1}{y^{2}}>\varepsilon=L>0 \text { for } y \neq 0,
$$

where $\varepsilon$ is a certain positive number. It is clear that assumptions $\left(H_{1}\right)-\left(H_{4}\right)$ hold.
Further from (22), we have

$$
\begin{aligned}
& u(\lambda)=\prod_{v=\lambda_{0}}^{\lambda-1} \frac{\psi(v)}{\psi(v)-p(v)}=\prod_{v=2}^{\lambda-1} \frac{v}{v-1}=\lambda-1 ; \\
& \Delta u(\lambda)=u(\lambda+1)-u(\lambda)=1 ; \\
& \Delta\left[\frac{a(\lambda)}{b(\lambda)}\right]=\Delta\left[\frac{\lambda^{2}}{\lambda}\right]=\Delta[\lambda] \neq 0 ; \\
& \lim _{\lambda \rightarrow \infty} \sum_{v=\lambda_{0}}^{\lambda-1} \frac{a(v)}{b(v)}=\lim _{\lambda \rightarrow \infty} \sum_{v=1}^{\lambda-1} \frac{v^{2}}{v}=\lim _{\lambda \rightarrow \infty} \sum_{v=\lambda_{0}}^{\lambda-1} v<\infty ; \\
& \lim _{\lambda \rightarrow \infty} \sum_{v=\lambda_{0}}^{\lambda-1}\left[\frac{1}{u(v) \psi(v)}\right]^{1 / \eta}=\lim _{\lambda \rightarrow \infty} \sum_{v=2}^{\lambda-1}\left[\frac{v}{(v-1)}\right]=\infty .
\end{aligned}
$$

We define the double sequence $H(\lambda, v)$ as follows:

(1) $H(\lambda, v)=(2 \lambda-v)^{2}>0$ for $\lambda>v>2$

(2) $H(\lambda, 2)=(\lambda-2)^{2}>0$ for $\lambda>s=2$

(3) $\Delta_{2} H(\lambda, v)=H(\lambda, v+1)-H(\lambda, v)=[2 \lambda-(v+1)]^{2}-$ $(2 \lambda-v)^{2}$

(4) $\Delta_{2} H(\lambda, v)=2 v-4 \lambda+1<0$ for $\lambda>v \geq 2$

and

$$
\begin{aligned}
h_{+}(\lambda, v) & =\Delta_{2} H(\lambda, v)+\frac{\Delta u(v) H(\lambda, v)}{u(v+1)} \\
& =\frac{\left(3 v^{2}-8 l v+v+4 \lambda^{2}\right)}{v} .
\end{aligned}
$$

Then

$$
\begin{gathered}
\frac{1}{H\left(\lambda, \lambda_{0}\right)} \sum_{v=\lambda_{2}}^{\lambda-1}\left[L u(v) q(v) H(\lambda, v)-\frac{h_{+}^{2}(\lambda, v) u(v+1) \psi(v+1) n_{0}^{\eta}}{4 H(\lambda, v)}\right] \\
=\frac{1}{(\lambda-2)^{2}} \sum_{v=\lambda_{2}}^{\lambda-1}\left[\frac{(2 \lambda-v)^{2}}{2}-\frac{\left(3 v^{2}-8 l v+v+4 \lambda^{2}\right)^{2}}{2(v+1)(2 \lambda-v)^{2}}\right],
\end{gathered}
$$

for $\lambda_{2}>\lambda_{1}$, and hence

$$
\begin{aligned}
& \lim _{\lambda \rightarrow \infty} \frac{1}{H\left(\lambda, \lambda_{0}\right)} \sum_{v=\lambda_{2}}^{\lambda-1}\left[L u(v) q(v) H(\lambda, v)-\frac{h_{+}^{2}(\lambda, v) u(v+1) \psi(v+1) n_{0}^{\eta}}{4 H(\lambda, v)}\right] \\
& \quad \lim _{\lambda \rightarrow \infty} \frac{1}{(\lambda-2)^{2}} \sum_{v=\lambda_{2}}^{\lambda-1}\left[\frac{(2 \lambda-v)^{2}}{2}-\frac{\left(3 v^{2}-8 l v+v+4 \lambda^{2}\right)^{2}}{2(v+1)(2 \lambda-v)^{2}}\right]=\infty .
\end{aligned}
$$

Therefore, condition (55) is satisfied. We deduce that all solutions of Eq. (84) are oscillatory by Theorem 7. 


\section{A Concluding Remark}

In this paper, we obtained new oscillation theorems for a class of fractional difference equation. The main outcomes are proved via the means of mathematical inequalities, properties of fractional operators, and generalized Riccati technique. We claim that the concluded results have merit and considered as an extension for the corresponding fractional differential equations. Particular examples that are consistent to the main results are demonstrated at the end of the paper.

The consideration of Eq. (1) with forcing term of the form

$$
\begin{aligned}
& \Delta\left[\psi(\lambda) z^{\eta}(\lambda)\right]+p(\lambda) z^{\eta}(\lambda)+q(\lambda) F \\
& \quad\left(\sum_{s=\lambda_{0}}^{\lambda-1+\mu}(\lambda-s-1)^{(-\mu)} y(s)\right)=g(\lambda),
\end{aligned}
$$

could be further investigated. We leave this for future consideration.

\section{Data Availability}

Data sharing not applicable to this article as no data sets were generated or analyzed during the current study.

\section{Conflicts of Interest}

The authors declare that they have no competing interests.

\section{Authors' Contributions}

The authors declare that the study was realized in collaboration with equal responsibility. All authors read and approved the final manuscript.

\section{Acknowledgments}

J. Alzabut would like to thank Prince Sultan University for supporting this work through the research group Nonlinear Analysis Methods in Applied Mathematics (NAMAM) group number RG-DES-2017-01-17.

\section{References}

[1] C. S. Goodrich and A. Peterson, Discrete Fractional Calculus, Springer, New York, 2015.

[2] F. N. Atici and P. W. Eloe, "A transform method in discrete fractional calculus," International Journal of Difference Equations, vol. 2, pp. 165-176, 2007.

[3] Y. Wang, Z. Han, P. Zhao, and S. Sun, "On the oscillation and asymptotic behavior for a kind of fractional differential equations," Advances in Difference Equations, vol. 2014, no. 1, 2014.

[4] S. Xiang, Z. Han, P. Zhao, and Y. Sun, "Oscillation behavior for a class of differential equation with fractional- order derivatives," Abstract and Applied Analysis, vol. 2014, Article ID 419597, 9 pages, 2014.

[5] R. L. Bagley and P. J. Torvik, "A theoretical basis for the application of fractional calculus to viscoelasticity," Journal of Rheology, vol. 27, no. 3, pp. 201-210, 1983.
[6] R. T. Baillie, "Long memory processes and fractional integration in econometrics," Journal of Econometrics, vol. 73, no. 1, pp. 5-59, 1996.

[7] M. Iswarya, R. Raja, G. Rajchakit, J. Cao, J. Alzabut, and C. Huang, "A perspective on graph theory based stability analysis of impulsive stochastic recurrent neural networks with time-varying delays," Advances in Difference Equations, vol. 2019, no. 1, 2019.

[8] B. Mandelbrot, "Some noises withI/fspectrum, a bridge between direct current and white noise," IEEE Transactions on Information Theory, vol. 13, no. 2, pp. 289-298, 1967.

[9] R. L. Magin, "Fractional calculus in bioengineering," Critical Reviews in Biomedical Engineering, vol. 32, no. 1, pp. 1-104, 2004.

[10] G. Rajchakit, A. Pratap, R. Raja, J. Cao, J. Alzabut, and C. Huang, "Hybrid control scheme for projective lag synchronization of Riemann-Liouville sense fractional order memristive BAM NeuralNetworks with mixed delays," Mathematics, vol. 7, no. 8, p. 759, 2019.

[11] Y. A. Rossikhin and M. V. Shitikova, "Applications of fractional calculus to dynamic problems of linear and nonlinear hereditary mechanics of solids," Applied Mechanics Reviews, vol. 50, no. 1, pp. 15-67, 1997.

[12] H. Zhou, J. Alzabut, and L. Yang, "On fractional Langevin differential equations with anti-periodic boundary conditions," European Physical Journal-Special Topics, vol. 226, pp. 35773590, 2017.

[13] T. Abdeljawad, "On delta and nabla Caputo fractional differences and dual identities," Discrete Dynamics in Nature and Society, vol. 2013, Article ID 406910, 12 pages, 2013.

[14] J. Alzabut, T. Abdeljawad, and D. Baleanu, "Nonlinear delay fractional difference equations with applications on discrete fractional Lotka-Volterra competition model," Journal of Computational Analysis and Applications, vol. 25, no. 5, pp. 889-898, 2018.

[15] J.-F. Cheng and Y.-M. Chu, "On the fractional difference equations of order (2,q)," Abstract and Applied Analysis, vol. 2011, Article ID 497259, 16 pages, 2011.

[16] T. Abdeljawad, F. Jarad, and J. Alzabut, "Fractional proportional differences with memory," The European Physical Journal Special Topics, vol. 226, no. 16-18, pp. 3333-3354, 2017.

[17] F. M. Atici and P. W. Eloe, "Initial value problems in discrete fractional calculus," Proceedings of the American Mathematical Society, vol. 137, no. 3, pp. 981-989, 2009.

[18] F. M. Atici and P. W. Eloe, "Linear systems of fractional nabla difference equations," Rocky Mountain Journal of Mathematics, vol. 41, no. 2, pp. 353-370, 2011.

[19] L. Erbe, C. S. Goodrich, B. Jia, and A. Peterson, "Survey of the qualitative properties of fractional difference operators: monotonicity, convexity, and asymptotic behavior of solutions," Advances in Difference Equations, vol. 2016, no. 1, 2016.

[20] C. S. Goodrich, "On a discrete fractional three-point boundary value problem," Journal of Difference Equations and Applications, vol. 18, no. 3, pp. 397-415, 2012.

[21] B. Abdalla, K. Abodayeh, T. Abdeljawad, and J. Alzabut, "New oscillation criteria for forced nonlinear fractional difference equationS," Vietnam Journal of Mathematics, vol. 45, no. 4, pp. 609-618, 2017.

[22] J. Alzabut, T. Abdeljawad, and H. Alrabaiah, "Oscillation criteria for forced and damped nable fractional difference 
equations," Journal of Computational Analysis and Applications, vol. 24, no. 8, pp. 1387-1394, 2018.

[23] G. E. Chatzarakis, A. G. M. Selvam, R. Janagaraj, and M. Douka, "Oscillation theorems for certain forced nonlinear discrete fractional order equations," Communications in Mathematics and Applications, vol. 10, no. 4, pp. 763-772, 2019.

[24] S. Kisalar, N. K. Yildiz, and E. Aktoprak, "Oscillation of higher order fractional nonlinear difference equations," International Journal of Difference Equations, vol. 10, no. 2, pp. 201-212, 2015.

[25] A. G. M. Selvam and R. Janagaraj, Oscillatory Behavior of Fractional Order Difference Equations with Damping, American International Journal of Research in Science, Technology, Engineering \& Mathematics, 2019.

[26] A. G. M. Selvam and R. Janagaraj, "Oscillation theorems for damped fractional order difference equations," AIP Conference Proceedings, vol. 2095, pp. 1-7, 2019.

[27] A. G. M. Selvam and R. Janagaraj, "Oscillation criteria of a class of fractional order damped difference equations," International Journal of Applied Mathematics, vol. 32, no. 3, pp. 433-441, 2019.

[28] W. N. Li, W. Sheng, and P. Zhang, "Oscillatory properties of certain non-linear fractional nabla difference equations," Journal of Applied Analysis and Computation, vol. 8, no. 6, pp. 1910-1918, 2016.

[29] J. Yang, A. Liu, and T. Liu, "Forced oscillation of nonlinear fractional differential equations with damping term," Advances in Difference Equations, vol. 2015, no. 1, 2015.

[30] S. Elaydi, "An introduction to difference equations," in Undergraduate Texts in Mathematics, Springer International Edition, 1996.

[31] Z. Bai and R. Xu, "The asymptotic behavior of solutions for a class of nonlinear fractional difference equations with damping term," Discrete Dynamics in Nature and Society, vol. 2018, Article ID 5232147, 11 pages, 2018.

[32] G. H. Hardy, J. E. Littlewood, and G. Polya, Inequalities, Cambridge University Press, Cambridge, 1959. 